\title{
Origin of single transverse-spin asymmetries in high-energy collisions
}

\author{
Justin Cammarota $\odot,{ }^{1,2, *}$ Leonard Gamberg $\oplus_{,}^{3, \dagger}$ Zhong-Bo Kang $\odot_{,, 5,6, \ddagger}^{4}$ Joshua A. Miller, ${ }^{2, \S}$ Daniel Pitonyak $\oplus^{2, \|}$ \\ Alexei Prokudin $\oplus^{3,7,9}$ Ted C. Rogers, ${ }^{9,8, *}$ and Nobuo Sato ${ }^{7, \dagger \dagger}$ \\ (Jefferson Lab Angular Momentum (JAM) Collaboration) \\ ${ }^{1}$ Physics Department, William \& Mary, Williamsburg, Virginia 23187, USA \\ ${ }^{2}$ Department of Physics, Lebanon Valley College, Annville, Pennsylvania 17003, USA \\ ${ }^{3}$ Division of Science, Penn State University Berks, Reading, Pennsylvania 19610, USA \\ ${ }^{4}$ Department of Physics and Astronomy, University of California, Los Angeles, California 90095, USA \\ ${ }^{5}$ Mani L. Bhaumik Institute for Theoretical Physics, University of California, \\ Los Angeles, California 90095, USA \\ ${ }^{6}$ Center for Frontiers in Nuclear Science, Stony Brook University, Stony Brook, New York 11794, USA \\ ${ }^{7}$ Thomas Jefferson National Accelerator Facility, Newport News, Virginia 23606, USA \\ ${ }^{8}$ Department of Physics, Old Dominion University, Norfolk, Virginia 23529, USA
}

(Received 19 February 2020; revised 27 May 2020; accepted 11 August 2020; published 8 September 2020)

\begin{abstract}
In this paper, we perform the first simultaneous QCD global analysis of data from semi-inclusive deep inelastic scattering, Drell-Yan, $e^{+} e^{-}$annihilation into hadron pairs, and proton-proton collisions. Consequently, we are able to extract a universal set of nonperturbative functions that describes the observed asymmetries in these reactions. The outcome of our analysis indicates single transverse-spin asymmetries in high-energy collisions have a common origin. Furthermore, we achieve the first phenomenological agreement with lattice QCD on the up and down quark tensor charges.
\end{abstract}

DOI: 10.1103/PhysRevD.102.054002

\section{INTRODUCTION}

For some 50 years, the spin and momentum structure of hadrons has been investigated in terms of their partonic (quark and gluon) content within the theory of QCD. Single transverse-spin asymmetries (SSAs) have played a central role in these studies. Early predictions from QCD that SSAs in single-inclusive hadron production should be exceedingly small [1] were in stark contrast with measurements showing large asymmetries [2,3] that persist in recent experiments [4-18].

A better understanding of SSAs has emerged with the aid of QCD factorization theorems [19-23]. They separate cross

\footnotetext{
*jcammarota@email.wm.edu lpg10@psu.edu zkang@physics.ucla.edu

¡jam017@lvc.edu

pitonyak@lvc.edu

prokudin@jlab.org

tcrogers@jlab.org

nsato@jlab.org
}

Published by the American Physical Society under the terms of the Creative Commons Attribution 4.0 International license. Further distribution of this work must maintain attribution to the author(s) and the published article's title, journal citation, and DOI. Funded by SCOAP ${ }^{3}$. sections into perturbatively calculable scattering contributions and nonperturbative physics encoded in parton distribution functions (PDFs) and fragmentation functions (FFs).

For processes with one large measured scale, $Q \gg$ $\Lambda_{\mathrm{QCD}}$, where $\Lambda_{\mathrm{QCD}}$ is a typical hadronic mass, experiments are sensitive to the collinear motion of partons. For example, in $p^{\uparrow} p \rightarrow h X$, the hard scale is set by the hadron transverse momentum $P_{h T}$. In this case, collinear twist-3 (CT3) factorization $[19,20]$ is valid, and spin asymmetries arise due to the quantum mechanical interference from multiparton states [19,20,24-33].

For reactions with two scales $Q_{2} \gg Q_{1} \sim \Lambda_{\mathrm{QCD}}$, experiments probe also intrinsic transverse parton motion. For example, in semi-inclusive lepton-nucleon deep inelastic scattering (SIDIS), $\ell N \rightarrow \ell h X$, one has $\Lambda_{\mathrm{QCD}} \sim P_{h T} \ll Q$, where $-Q^{2}$ is the photon virtuality. For such processes, transverse momentum dependent (TMD) factorization [21-23,34,35] is valid, and the mechanism responsible for spin asymmetries is encoded in TMD PDFs and FFs (collectively called TMDs) [36-41].

There are theoretical calculations that use CT3 and TMD factorization theorems to yield a unified picture of spin asymmetries in hard processes [42-47]. This is one of the cornerstones for studying the three-dimensional structure of hadrons at existing [48-52] and future facilities, 
including the Electron-Ion Collider [53,54]. In this paper, we provide, for the first time, phenomenological results that indicate SSAs have a common origin. We perform the first simultaneous QCD global analysis of the available data in SIDIS, Drell-Yan (DY), semi-inclusive $e^{+} e^{-}$annihilation (SIA), and proton-proton collisions. Furthermore, we find, for the first time, excellent agreement with lattice QCD for the up and down quark tensor charges.

\section{THEORETICAL BACKGROUND}

The key observation that makes our analysis possible is that in both the CT3 and TMD formalisms, collinear multiparton correlations play an important role. A generic TMD PDF $F\left(x, k_{T}\right)$ depends on $x$, the fraction of the nucleon's longitudinal momentum carried by the parton, and $k_{T} \equiv\left|\vec{k}_{T}\right|$, the parton's transverse momentum. The same TMD when Fourier conjugated into position $\left(b_{T}\right)$ space [35,55-57] exhibits an operator product expansion (OPE) in the limit when $b_{T}$ is small. TMDs relevant for SSAs can be expressed in terms of CT3 multiparton correlation functions in this OPE [57-60].

Another way to establish the connection between CT3 functions and TMDs is by the use of parton model identities. One such relation, derived at the level of QCD operators, is [61]

$$
\pi F_{F T}(x, x)=\int d^{2} \vec{k}_{T} \frac{k_{T}^{2}}{2 M^{2}} f_{1 T}^{\perp}\left(x, k_{T}^{2}\right) \equiv f_{1 T}^{\perp(1)}(x),
$$

where $F_{F T}(x, x)$ is the Qiu-Sterman CT3 matrix element and $f_{1 T}^{\perp(1)}(x)$ is the first moment of the TMD Sivers function $f_{1 T}^{\perp}\left(x, k_{T}^{2}\right)[62,63]$. Here, we do not address the validity of this relation beyond leading order [57-60,64].

A central focus of TMD asymmetries has been on the Sivers and Collins SSAs in SIDIS, $A_{U T}^{\sin \left(\phi_{h}-\phi_{S}\right)} \equiv A_{\text {SIDIS }}^{\text {Siv }}[65-$ 70] and $A_{U T}^{\sin \left(\phi_{h}+\phi_{S}\right)} \equiv A_{\text {SIDIS }}^{\mathrm{Col}}[66-69,71]$; Sivers SSA in DY, $A_{\mathrm{DY}}^{\mathrm{Siv}}$, for $W^{ \pm} / Z$ production $\equiv A_{N}^{W / Z}$ [72] and for $\mu^{+} \mu^{-}$ production $\equiv A_{T, \mu^{+} \mu^{-}}^{\sin \phi_{S}}$ [73]; and Collins SSA in SIA, $A_{\mathrm{SIA}}^{\mathrm{Col}}$ [74-78]. The relevant TMDs probed by these processes [36-41] are the transversity TMD $h_{1}\left(x, k_{T}^{2}\right)$ [79], the Sivers function $f_{1 T}^{\perp}\left(x, k_{T}^{2}\right) \quad[62,63]$, and Collins function $H_{1}^{\perp}\left(z, z^{2} p_{\perp}^{2}\right)$ [80]. Each of them can be written in a model-independent way in terms of a collinear counterpart using the OPE. The function $h_{1}\left(x, k_{T}^{2}\right)$ is related to the collinear (twist-2) transversity function $h_{1}(x)$ [81], $f_{1 T}^{\perp}\left(x, k_{T}^{2}\right)$ to the Qiu-Sterman function $F_{F T}(x, x)$ [57], and $H_{1}^{\perp}\left(z, z^{2} p_{\perp}^{2}\right)$ to its first $p_{\perp}$-moment [82], defined as

$$
H_{1}^{\perp(1)}(z) \equiv z^{2} \int d^{2} \vec{p}_{\perp} \frac{p_{\perp}^{2}}{2 M_{h}^{2}} H_{1}^{\perp}\left(z, z^{2} p_{\perp}^{2}\right),
$$

where $M_{h}$ is the hadron mass and $p_{\perp}$ is the parton transverse momentum. Note $H_{1}^{\perp(1)}(z)$ is a CT3 function (the so-called kinematical type [58]).
The same set of functions, $h_{1}(x), F_{F T}(x, x), H_{1}^{\perp(1)}(z)$ in the OPE of TMDs are also the nonperturbative objects that drive the collinear SSA $A_{N}^{h}$ in $p^{\uparrow} p \rightarrow h X[26,28,30-33]$. In fact, in the CT3 framework, the main cause of $A_{N}^{h}$ can be explained by the coupling of $h_{1}(x)$ to $H_{1}^{\perp(1)}(z)$ and another multiparton correlator $\tilde{H}(z)[83,84]$. The latter generates the $P_{h T}$-integrated SIDIS $A_{U T}^{\sin \phi_{S}}$ asymmetry by coupling with $h_{1}(x)$ [39]. In $A_{N}^{h}$, we include both the Qiu-Sterman ("Sivers-type") and fragmentation ("Collins-type") terms in our analysis. As in Refs. [83,84], we again find the former is negligible while the latter is dominant. Based on the above discussion, one can argue that SSAs have a common origin, namely, multiparton correlations.

We present, for the first time, a phenomenological verification of this by performing a simultaneous QCD global analysis of $A_{\mathrm{SIDIS}}^{\mathrm{Siv}}, A_{\mathrm{SIDIS}}^{\mathrm{Col}}, A_{\mathrm{DY}}^{\mathrm{Siv}}, A_{\mathrm{SIA}}^{\mathrm{Col}}$, and $A_{N}^{h}$. In addition, the fact that we are able to describe both $A_{\text {SIDIS }}^{\text {Siv }}$ and $A_{N}^{h}$ (where the latter includes both Collins-type and Sivers-type contributions) further indicates a resolution to the "sign-mismatch" puzzle between the Sivers function and Qiu-Sterman function [31] found when using the parton model relation, Eq. (1).

We further claim that such an analysis serves as a universality test since 1) the system must be overconstrained, i.e., the number of equations relating partonic functions to observables must be larger than the number of partonic functions; 2) each function must appear at least twice in such equations; and 3) there must be reasonable kinematical overlap between observables. These conditions are satisfied in our analysis, as summarized in Table I. There is also considerable kinematical overlap in $x, z$, and $Q^{2}$ between observables. SIDIS covers a region $x \lesssim 0.3$, $0.2 \lesssim z \lesssim 0.6$, and $2 \lesssim Q^{2} \lesssim 40 \mathrm{GeV}^{2}$. SIA data have $0.2 \lesssim z \lesssim 0.8$ and $Q^{2} \approx 13 \mathrm{GeV}^{2}$ or $110 \mathrm{GeV}^{2}$. For DY data, $0.1 \lesssim x \lesssim 0.35$ and $Q^{2} \approx 30 \mathrm{GeV}^{2}$ or $(80 \mathrm{GeV})^{2}$. Lastly, $A_{N}^{h}$ integrates from $x_{\min }$ to 1 and $z_{\min }$ to 1 . For $A_{N}^{\pi^{ \pm}}$data from BRAHMS, $0.2 \lesssim\left(x_{\min }, z_{\text {min }}\right) \lesssim 0.3$, with $1 \lesssim Q^{2} \lesssim 6 \mathrm{GeV}^{2}$. The $A_{N}^{\pi^{0}}$ data from STAR have $0.2 \lesssim\left(x_{\min }, z_{\min }\right) \lesssim 0.7$, and $1 \lesssim Q^{2} \lesssim 13 \mathrm{GeV}^{2}$. Moreover, we provide additional evidence in Sec. IV that SSAs for TMD and CT3 observables have a common origin by first extracting the TMDs from only SSAs in SIDIS, DY, and $e^{+} e^{-}$and then making predictions for $A_{N}^{\pi}$ based on those results. A necessary condition for TMD and CT3 SSAs to have the same dynamical origin is that, within error bands, our predictions should describe the $A_{N}^{\pi}$ measurements. Indeed, this is exactly what we find, as we will show later in Sec. IV.

\section{METHODOLOGY}

To perform our global analysis, we must postulate a functional form for the nonperturbative functions. For the TMDs, we decouple the $x$ and $k_{T}$ ( $z$ and $p_{\perp}$ ) dependence. 
TABLE I. Summary of the SSAs analyzed in our global fit. There are a total of 18 different reactions. (UC and UL stand for "unlikecharged" and "unlike-like" pion combinations.) There is also a total of 6 nonperturbative functions when one takes into account flavor separation.

\begin{tabular}{|c|c|c|c|c|}
\hline Observable & Reactions & Nonperturbative function(s) & $\chi^{2} / N_{\text {pts. }}$. & Refs. \\
\hline$A_{\text {SIDIS }}^{\text {Siv }}$ & $e+(p, d)^{\uparrow} \rightarrow e+\left(\pi^{+}, \pi^{-}, \pi^{0}\right)+X$ & $f_{1 T}^{\perp}\left(x, k_{T}^{2}\right)$ & $150.0 / 126=1.19$ & {$[65,66,68]$} \\
\hline$A_{\mathrm{SIDIS}}^{\mathrm{Col}}$ & $e+(p, d)^{\uparrow} \rightarrow e+\left(\pi^{+}, \pi^{-}, \pi^{0}\right)+X$ & $h_{1}\left(x, k_{T}^{2}\right), H_{1}^{\perp}\left(z, z^{2} p_{\perp}^{2}\right)$ & $111.3 / 126=0.88$ & {$[66,68,71]$} \\
\hline$A_{\text {SIA }}^{\mathrm{Col}}$ & $e^{+}+e^{-} \rightarrow \pi^{+} \pi^{-}(U C, U L)+X$ & $H_{1}^{\perp}\left(z, z^{2} p_{\perp}^{2}\right)$ & $154.5 / 176=0.88$ & {$[74-77]$} \\
\hline$A_{\mathrm{DY}}^{\mathrm{Siv}}$ & $\pi^{-}+p^{\uparrow} \rightarrow \mu^{+} \mu^{-}+X$ & $f_{1 T}^{\perp}\left(x, k_{T}^{2}\right)$ & $5.96 / 12=0.50$ & [73] \\
\hline$A_{\mathrm{DY}}^{\mathrm{Si}}$ & $p^{\uparrow}+p \rightarrow\left(W^{+}, W^{-}, Z\right)+X$ & $f_{1 T}^{\perp}\left(x, k_{T}^{2}\right)$ & $31.8 / 17=1.87$ & [72] \\
\hline$A_{N}^{h}$ & $p^{\uparrow}+p \rightarrow\left(\pi^{+}, \pi^{-}, \pi^{0}\right)+X$ & $h_{1}(x), F_{F T}(x, x)=\frac{1}{\pi} f_{1 T}^{\perp(1)}(x), H_{1}^{\perp(1)}(z)$ & $66.5 / 60=1.11$ & {$[7,9,10,13]$} \\
\hline
\end{tabular}

This is phenomenologically well motivated within the literature and has been successfully used in a wide variety of reactions-see, e.g., Refs. [85-100]. This ansatz is also supported by a lattice QCD calculation in Ref. [101]. We employ a Gaussian parametrization for the transverse momentum dependence. This assumes most of the transverse momentum is nonperturbative and thus related to intrinsic properties of the colliding hadrons rather than to hard gluon radiation.

Although this type of parametrization does not have the complete features of TMD evolution, it was shown in Refs. [102,103] that utilizing such a parametrization is comparable to full TMD evolution at next-to-leadinglogarithmic accuracy [82,104-107]. In addition, asymmetries are ratios of cross sections where evolution and next-to-leading-order effects tend to cancel out [107]. We also implement a Dokshitzer-Gribov-Lipatov-AltarelliParisi (DGLAP)-type evolution for the collinear twist-3 functions analogous to Ref. [108], where a doublelogarithmic $Q^{2}$-dependent term is explicitly added to the parameters. For the collinear twist-2 PDFs and FFs, we use the standard leading-order DGLAP evolution.

For the unpolarized and transversity TMDs, we have

$$
f^{q}\left(x, k_{T}^{2}\right)=f^{q}(x) \mathcal{G}_{f}^{q}\left(k_{T}^{2}\right),
$$

where the generic function $f=f_{1}$ or $h_{1}$, and

$$
\mathcal{G}_{f}^{q}\left(k_{T}^{2}\right)=\frac{1}{\pi\left\langle k_{T}^{2}\right\rangle_{f}^{q}} \exp \left[-\frac{k_{T}^{2}}{\left\langle k_{T}^{2}\right\rangle_{f}^{q}}\right]
$$

Using the relation $\pi F_{F T}(x, x)=f_{1 T}^{\perp(1)}(x)$ [61], the Sivers function reads

$$
f_{1 T}^{\perp q}\left(x, k_{T}^{2}\right)=\frac{2 M^{2}}{\left\langle k_{T}^{2}\right\rangle_{f_{1 T}^{\perp}}^{q}} \pi F_{F T}(x, x) \mathcal{G}_{f_{1 T}^{\perp}}^{q}\left(k_{T}^{2}\right) .
$$

For the TMD FFs, the unpolarized function is parametrized as

$$
D_{1}^{h / q}\left(z, z^{2} p_{\perp}^{2}\right)=D_{1}^{h / q}(z) \mathcal{G}_{D_{1}}^{h / q}\left(z^{2} p_{\perp}^{2}\right),
$$

while the Collins FF reads

$$
H_{1}^{\perp h / q}\left(z, z^{2} p_{\perp}^{2}\right)=\frac{2 z^{2} M_{h}^{2}}{\left\langle P_{\perp}^{2}\right\rangle_{H_{1}^{\perp}}^{h / q}} H_{1 h / q}^{\perp(1)}(z) \mathcal{G}_{H_{1}^{\perp}}^{h / q}\left(z^{2} p_{\perp}^{2}\right),
$$

where we have explicitly written its $z$ dependence in terms of its first moment $H_{1 h / q}^{\perp(1)}(z)$ [82]. The widths for the FFs are denoted as $\left\langle P_{\perp}^{2}\right\rangle_{D}^{h / q}$, where $D=D_{1}$ or $H_{1}^{\perp}$. (Note that the hadron transverse momentum $\vec{P}_{\perp}=-z \vec{p}_{\perp}$.) For $f_{1}^{q}(x)$ and $D_{1}^{q}(z)$, we use the leading-order CTEQ-Jefferson Lab [109] and de Florian-Sassot-Stratmann [110] functions. The pion PDFs are taken from Ref. [111] and are next-to-leading order [112].

Note Eqs. (3), (5), and (7) make clear that the underlying nonperturbative functions, $h_{1}(x), F_{F T}(x, x), H_{1}^{\perp(1)}(z)$, that drive the (TMD) SSAs $A_{\mathrm{SIDIS}}^{\mathrm{Siv}}, A_{\mathrm{SIDIS}}^{\mathrm{Col}}, A_{\mathrm{DY}}^{\mathrm{Siv}}$, and $A_{\mathrm{SIA}}^{\mathrm{Col}}$, are the same collinear functions that enter the SSA $A_{N}^{h}$ [along with $\tilde{H}(z)$ ]. We generically parametrize these collinear functions as

$F^{q}(x)=\frac{N_{q} x^{a_{q}}(1-x)^{b_{q}}\left(1+\gamma_{q} x^{\alpha_{q}}(1-x)^{\beta_{q}}\right)}{\mathrm{B}\left[a_{q}+2, b_{q}+1\right]+\gamma_{q} \mathrm{~B}\left[a_{q}+\alpha_{q}+2, b_{q}+\beta_{q}+1\right]}$,

where $F^{q}=h_{1}^{q}, \pi F_{F T}^{q}, H_{1 h / q}^{\perp(1)}$ (with $x \rightarrow z$ for the Collins function) and $B$ is the Euler beta function. In the course of our analysis, we found that $\tilde{H}(z)$ was consistent with zero within error bands. Therefore, data on the aforementioned ( $P_{h T}$-integrated) $A_{U T}^{\sin \phi_{S}}$ asymmetry in SIDIS is needed to properly constrain $\tilde{H}(z)$. For now, we set $\tilde{H}(z)$ to zero, which is consistent with preliminary data from HERMES [113] and COMPASS [114] showing a small $A_{U T}^{\sin \phi_{S}}$.

For the collinear PDFs $h_{1}^{q}(x)$ and $\pi F_{F T}^{q}(x, x)$, we only allow $q=u, d$ and set antiquark functions to zero. For both functions, $\{\gamma, \alpha, \beta\}$ are not used, and we set $b_{u}=b_{d}$. This approach is similar to previous analyses $[82,95,102$, 
$103,106,115]$. For the collinear FF $H_{1 h / q}^{\perp(1)}(z)$, we allow for favored (fav) and unfavored (unf) parameters. We also found that, similar to what has been done in fits of unpolarized collinear FFs [110], $\{\gamma, \beta\}$ are needed for $H_{1 h / q}^{\perp(1)}(z)$, while $\alpha$ can be set to zero since $A_{\mathrm{SIA}}^{\mathrm{Col}}, A_{\mathrm{SIDIS}}^{\mathrm{Col}}$ are at $z \gtrsim 0.2$. The need for $\{\gamma, \beta\}$ is due to the fact that the data for $A_{\mathrm{SIA}}^{\mathrm{Col}}$ have a different shape at smaller versus larger $z$. Indeed, we found that $\left(\chi^{2} / N_{\text {pts. }}\right)_{\text {SIA }}=3.85$ if $H_{1 h / q}^{\perp(1)}(z)$ only has a functional form proportional to $N z^{a}(1-z)^{b}$. In the end, we have a total of 20 parameters for the collinear functions. There are also four parameters for the transverse momentum widths associated with $h_{1}, f_{1 T}^{\perp}$, and $H_{1}^{\perp}:\left\langle k_{T}^{2}\right\rangle_{f_{1 T}^{\perp}}^{u}=$ $\left\langle k_{T}^{2}\right\rangle_{f_{1 T}^{\perp}}^{d} \equiv\left\langle k_{T}^{2}\right\rangle_{f_{1 T}^{\perp}},\left\langle k_{T}^{2}\right\rangle_{h_{1}}^{u}=\left\langle k_{T}^{2}\right\rangle_{h_{1}}^{d} \equiv\left\langle k_{T}^{2}\right\rangle_{h_{1}},\left\langle P_{\perp}^{2}\right\rangle_{H_{1}^{\perp}}^{f a v}$, and $\left\langle P_{\perp}^{2}\right\rangle_{H_{1}^{\perp}}^{\text {unf }}$

We extract unpolarized TMD widths $[85,96,97]$ by including HERMES pion and kaon multiplicities [116], which involves six more parameters: $\left\langle k_{T}^{2}\right\rangle_{f_{1}}^{v a l},\left\langle k_{T}^{2}\right\rangle_{f_{1}}^{\text {sea }}$, $\left\langle P_{\perp}^{2}\right\rangle_{D_{1}^{\{r, K\}}}^{f a v},\left\langle P_{\perp}^{2}\right\rangle_{D_{1}^{\{\pi, K\}}}^{u n f}$. The pion PDF widths are taken to be the same as those for the proton. We also include normalization parameters for each dataset to account for correlated systematic uncertainties.

We use the multistep strategy in a Monte Carlo framework developed in Ref. [117] to reliably sample the Bayesian posterior distribution for the parameters. This approach allows us to determine the relevant regions in parameter space and give state-of-the-art uncertainty quantification, for the hadronic structures that best describe the data.

\section{PHENOMENOLOGICAL RESULTS}

We first test the universality of our proposed mechanism by making predictions for $A_{N}^{\pi}$ using TMDs extracted from only SSAs in SIDIS, DY, and $e^{+} e^{-}$. The results are shown in Fig. 1 and are similar to what was found in Ref. [84]. As one can see, both the BRAHMS and STAR data fall within the theoretical predictions. The large uncertainties of the STAR predictions are due to the fact that the $x$-dependent PDFs (transversity and Qiu-Sterman) must be extrapolated beyond where they are constrained by the TMD SSAs. By
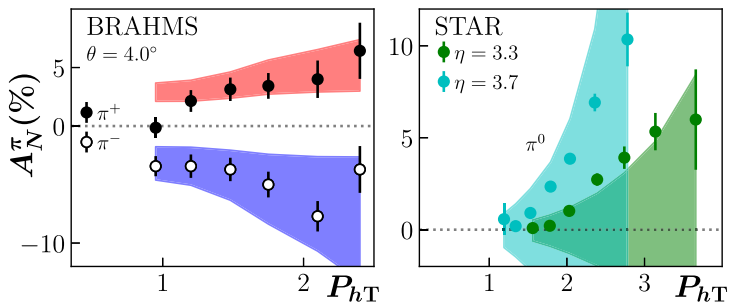

FIG. 1. Predictions for $A_{N}^{\pi}$ using TMDs extracted from only $A_{\mathrm{SIDIS}}^{\mathrm{Siv}}, A_{\mathrm{SIDIS}}^{\mathrm{Col}}, A_{\mathrm{DY}}^{\mathrm{Siv}}$, and $A_{\mathrm{SIA}}^{\mathrm{Col}}$. Similar results are found for the other BRAHMS and STAR datasets.
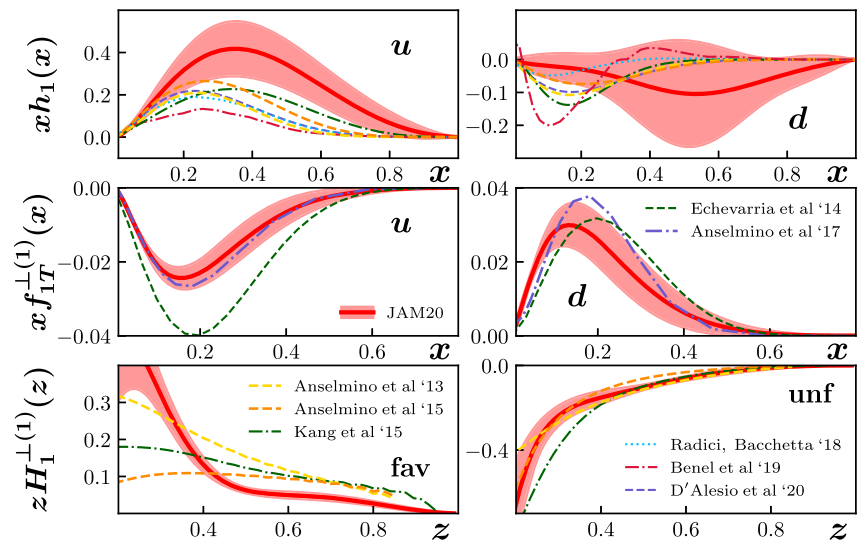

FIG. 2. The extracted functions $h_{1}(x), f_{1 T}^{\perp(1)}(x)$, and $H_{1}^{\perp(1)}(z)$ at $Q^{2}=4 \mathrm{GeV}^{2}$ from our (JAM20) global analysis (red solid curves with 1- $\sigma$ CL error bands). The functions from other groups $[82,95,102,103,106,115,119,120]$ are also shown.

including $A_{N}^{\pi}$ data in a simultaneous QCD global analysis of SSAs, we can decrease the theoretical error bands and isolate the PDF and FF solutions that optimize the description of all measurements.

We also emphasize that the number of parameters and functional form used in this fit, as described in Sec. III, do not guarantee one would be able to successfully describe all SSA data simultaneously. In general, we are interested in whether certain functions (transversity, Qiu-Sterman, and Collins first moment) have universal values for a given kinematic point irrespective of the process in which they are used. The answer to this question should be independent of how the functions are parametrized. In addition, if our parametrization were too flexible to where we overfit the data, one would expect poor predictions for $A_{N}^{\pi}$ in Fig. 1, which is not the case. Note that if the $A_{N}^{\pi}$ data did not fall within the predictions of Fig. 1, one would not expect to simultaneously describe all SSA data. We stress no additional parameters are introduced when $A_{N}^{\pi}$ is included in the combined analysis with TMD SSAs.

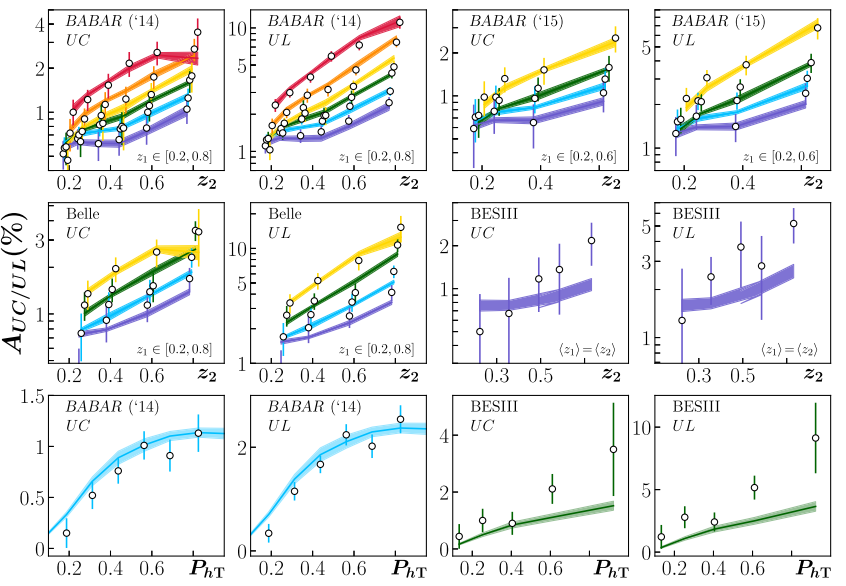

FIG. 3. Theory compared to experiment for $A_{\text {SIA }}^{\mathrm{Col}}$. 


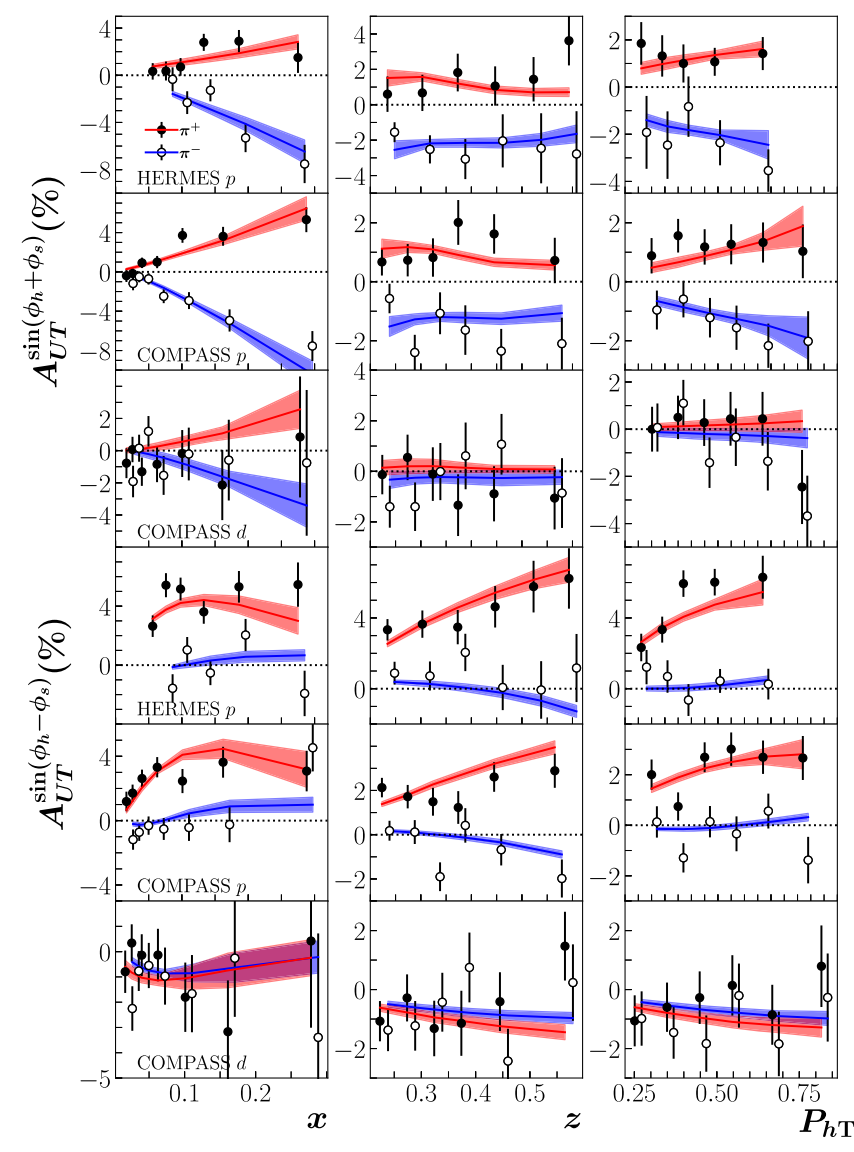

FIG. 4. Theory compared to experiment for $A_{\mathrm{SIDIS}}^{\mathrm{Col} / \mathrm{Siv}}$.

We now perform our simultaneous QCD global analysis of the SSA data summarized in Table I. The standard cuts of $0.2<z<0.6, Q^{2}>1.63 \mathrm{GeV}^{2}$, and $0.2<P_{h T}<$ $0.9 \mathrm{GeV}$ have been applied to all SIDIS datasets [97], and $P_{h T}>1 \mathrm{GeV}$ has been applied to all $A_{N}^{\pi}$ datasets [83,84], giving us a total of $517 \mathrm{SSA}$ data points in the fit
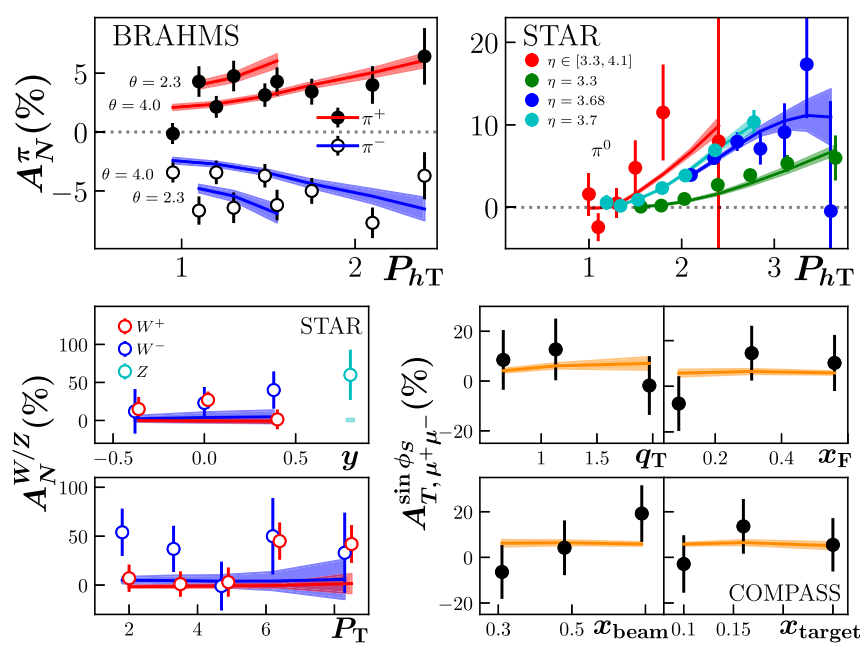

FIG. 5. Theory compared to experiment for $A_{N}^{\pi}$ and $A_{\mathrm{DY}}^{\mathrm{Siv}}$.

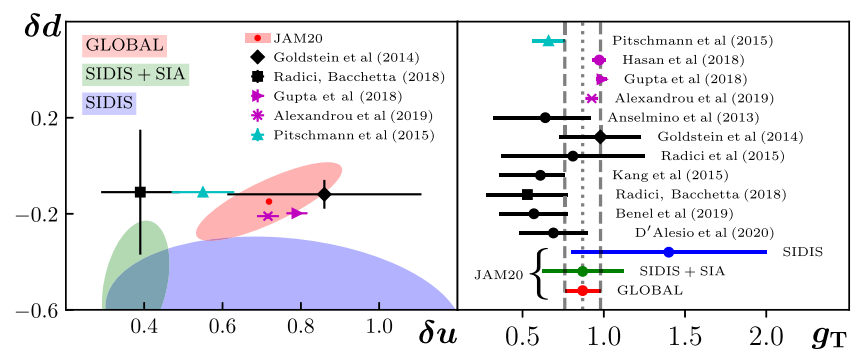

FIG. 6. The tensor charges $\delta u, \delta d$, and $g_{T}$. Our (JAM20) results at $Q^{2}=4 \mathrm{GeV}^{2}$ along with others from phenomenology (black), lattice QCD (purple), and Dyson-Schwinger (cyan).

along with 807 HERMES multiplicity [116] data points. The extracted functions [118] and their comparison to other groups are shown in Fig. 2. We obtain a good agreement between theory and experiment, as one sees in Figs. 3-5. Specifically, we find $\left(\chi^{2} / N_{\text {pts. }}\right)_{\text {SSA }}=520 / 517=1.01$ for SSA data alone, and $\chi^{2} / N_{\text {pts. }}=1373 / 1324=1.04$ for all data, including HERMES multiplicities.

Figure 6 displays our extracted tensor charges of the nucleon. The individual flavor charges $\delta q \equiv \int_{0}^{1} d x\left[h_{1}^{q}(x)-\right.$ $\left.h_{1}^{\bar{q}}(x)\right]$ are shown along with the isovector combination $g_{T} \equiv \delta u-\delta d$. We compare our results to those from lattice QCD computations at the physical point [121-123], other phenomenological extractions [82,95,115,119,120,124,125], and a calculation using Dyson-Schwinger equations [126]. From Fig. 6, the strong impact of including more SSA datasets is clear, highlighting the importance of carrying out a simultaneous extraction of partonic functions in a global analysis. In going from SIDIS $\rightarrow$ (SIDIS + SIA $) \rightarrow$ GLOBAL (where GLOBAL in particular includes $A_{N}^{\pi}$ ), we find $g_{T}=1.4(6) \rightarrow 0.87(25) \rightarrow 0.87(11)$. This is the most precise phenomenological determination of $g_{T}$ to date. All of the inferred tensor charges $\left(\delta u, \delta d\right.$, and $\left.g_{T}\right)$ are in excellent agreement with lattice QCD data. As can be seen from Fig. 6, including $A_{N}^{\pi}$ is crucial to achieve the agreement between our results $\delta u=0.72(19), \delta d=-0.15(16)$ and those from lattice QCD.

\section{CONCLUSIONS}

In this paper, we have performed the first simultaneous QCD global analysis of the available SSA data in SIDIS, $\mathrm{DY}, e^{+} e^{-}$annihilation, and proton-proton collisions. The predictive power exhibited by the results of the combined analysis indicates SSAs have a common origin. Namely, they are due to the intrinsic quantum-mechanical interference from multiparton states. Our findings imply that the effects are predominantly nonperturbative and intrinsic to hadronic wave functions. Also, the extracted up and down quark tensor charges are in excellent agreement with lattice QCD. 
The future data from JLab-12 GeV [51], COMPASS [49,50], an upgraded Relativistic Heavy Ion Collider [48], Belle II [52], and the Electron-Ion Collider [53,54] will help to reduce the uncertainties of the extracted functions. Measurements that have kinematical overlap to the current data, like SIDIS data from JLab-12 GeV [51,127] and an Electron-Ion Collider [54], more precise Drell-Yan data from COMPASS [49,50] and STAR [48], and new $A_{N}$ and pion-in-jet data from STAR [48], will test our results. Ultimately, all these measurements will lead to a deeper understanding of hadronic structure.

\section{ACKNOWLEDGMENTS}

This work has been supported by the NSF under Grants No. PHY-2012002 (A. P.), No. PHY-2011763 (D. P.), No. PHY-1720486 (Z. K.), and No. PHY-1945471 (Z. K.), the U.S. Department of Energy, under Contracts No. DE-FG02-07ER41460 (L. G.), No. DE-AC0506OR23177 (A.P., N.S., and T.R.) under which Jefferson Science Associates, LLC, manages and operates Jefferson Lab, a Lebanon Valley College (LVC) Arnold Student-Faculty Research Grant (J. A. M. and D. P.), and within the framework of the TMD Topical Collaboration.
[1] G. L. Kane, J. Pumplin, and W. Repko, Phys. Rev. Lett. 41, 1689 (1978).

[2] G. Bunce et al., Phys. Rev. Lett. 36, 1113 (1976).

[3] R. D. Klem, J. E. Bowers, H. W. Courant, H. Kagan, M. L. Marshak, E. A. Peterson, K. Ruddick, W. H. Dragoset, and J. B. Roberts, Phys. Rev. Lett. 36, 929 (1976).

[4] D. L. Adams et al. (E581 Collaboration), Phys. Lett. B 261, 201 (1991).

[5] K. Krueger et al., Phys. Lett. B 459, 412 (1999).

[6] C. E. Allgower et al., Phys. Rev. D 65, 092008 (2002).

[7] J. Adams et al. (STAR Collaboration), Phys. Rev. Lett. 92, 171801 (2004).

[8] S. S. Adler et al. (PHENIX Collaboration), Phys. Rev. Lett. 95, 202001 (2005).

[9] J. H. Lee and F. Videbaek (BRAHMS Collaboration), AIP Conf. Proc. 915, 533 (2007).

[10] B. I. Abelev et al. (STAR Collaboration), Phys. Rev. Lett. 101, 222001 (2008).

[11] I. Arsene et al. (BRAHMS Collaboration), Phys. Rev. Lett. 101, 042001 (2008).

[12] L. Adamczyk et al. (STAR Collaboration), Phys. Rev. D 86, 032006 (2012).

[13] L. Adamczyk et al. (STAR Collaboration), Phys. Rev. D 86, 051101 (2012).

[14] L. Bland et al. (AnDY Collaboration), Phys. Lett. B 750, 660 (2015).

[15] A. Adare et al. (PHENIX Collaboration), Phys. Rev. D 90, 012006 (2014).

[16] A. Adare et al. (PHENIX Collaboration), Phys. Rev. D 90, 072008 (2014).

[17] A. Airapetian et al. (HERMES Collaboration), Phys. Lett. B 728, 183 (2014).

[18] K. Allada et al. (Jefferson Lab Hall A Collaboration), Phys. Rev. C 89, 042201 (2014).

[19] J.-W. Qiu and G. Sterman, Phys. Rev. Lett. 67, 2264 (1991).

[20] J.-W. Qiu and G. Sterman, Nucl. Phys. B378, 52 (1992).

[21] J. C. Collins and D. E. Soper, Nucl. Phys. B193, 381 (1981).

[22] J. C. Collins, D. E. Soper, and G. Sterman, Nucl. Phys. B250, 199 (1985).
[23] R. Meng, F. I. Olness, and D. E. Soper, Phys. Rev. D 54, 1919 (1996).

[24] A. V. Efremov and O. V. Teryaev, Sov. J. Nucl. Phys. 36, 140 (1982).

[25] A. Efremov and O. Teryaev, Phys. Lett. B 150, 383 (1985).

[26] J.-W. Qiu and G. Sterman, Phys. Rev. D 59, 014004 (1998).

[27] H. Eguchi, Y. Koike, and K. Tanaka, Nucl. Phys. B752, 1 (2006).

[28] C. Kouvaris, J.-W. Qiu, W. Vogelsang, and F. Yuan, Phys. Rev. D 74, 114013 (2006).

[29] H. Eguchi, Y. Koike, and K. Tanaka, Nucl. Phys. B763, 198 (2007).

[30] Y. Koike and T. Tomita, Phys. Lett. B 675, 181 (2009).

[31] Z.-B. Kang, J.-W. Qiu, W. Vogelsang, and F. Yuan, Phys. Rev. D 83, 094001 (2011).

[32] A. Metz and D. Pitonyak, Phys. Lett. B 723, 365 (2013).

[33] H. Beppu, K. Kanazawa, Y. Koike, and S. Yoshida, Phys. Rev. D 89, 034029 (2014).

[34] X.-d. Ji, J.-P. Ma, and F. Yuan, Phys. Lett. B 597, 299 (2004).

[35] J. Collins, Cambridge Monogr. Part. Phys., Nucl. Phys., Cosmol. 32, 1 (2011).

[36] A. Kotzinian, Nucl. Phys. B441, 234 (1995).

[37] P. J. Mulders and R. D. Tangerman, Nucl. Phys. B461, 197 (1996).

[38] D. Boer, R. Jakob, and P. J. Mulders, Nucl. Phys. B504, 345 (1997).

[39] A. Bacchetta, M. Diehl, K. Goeke, A. Metz, P. J. Mulders et al., J. High Energy Phys. 02 (2007) 093.

[40] S. Arnold, A. Metz, and M. Schlegel, Phys. Rev. D 79, 034005 (2009).

[41] D. Pitonyak, M. Schlegel, and A. Metz, Phys. Rev. D 89, 054032 (2014).

[42] X. Ji, J.-W. Qiu, W. Vogelsang, and F. Yuan, Phys. Rev. Lett. 97, 082002 (2006).

[43] X. Ji, J.-W. Qiu, W. Vogelsang, and F. Yuan, Phys. Lett. B 638, 178 (2006).

[44] Y. Koike, W. Vogelsang, and F. Yuan, Phys. Lett. B 659 , 878 (2008). 
[45] J. Zhou, F. Yuan, and Z.-T. Liang, Phys. Rev. D 78, 114008 (2008).

[46] F. Yuan and J. Zhou, Phys. Rev. Lett. 103, 052001 (2009).

[47] J. Zhou, F. Yuan, and Z.-T. Liang, Phys. Rev. D 81, 054008 (2010).

[48] E.-C. Aschenauer et al., arXiv:1501.01220.

[49] F. Gautheron et al. (COMPASS Collaboration), Report No. CERN-SPSC-2010-014, 2010.

[50] F. Bradamante (COMPASS Collaboration), Proc. Sci., SPIN2018 (2018) 045.

[51] J. Dudek, R. Ent, R. Essig, K. Kumar, C. Meyer et al., Eur. Phys. J. A 48, 187 (2012).

[52] W. Altmannshofer et al. (Belle-II Collaboration), Prog. Theor. Exp. Phys. (2019), 123C01.

[53] D. Boer, M. Diehl, R. Milner, R. Venugopalan, W. Vogelsang et al., arXiv:1108.1713.

[54] A. Accardi et al., Eur. Phys. J. A 52, 268 (2016).

[55] J. C. Collins and D. E. Soper, Nucl. Phys. B194, 445 (1982).

[56] D. Boer, L. Gamberg, B. Musch, and A. Prokudin, J. High Energy Phys. 10 (2011) 021.

[57] S. M. Aybat, J. C. Collins, J.-W. Qiu, and T. C. Rogers, Phys. Rev. D 85, 034043 (2012).

[58] K. Kanazawa, Y. Koike, A. Metz, D. Pitonyak, and M. Schlegel, Phys. Rev. D 93, 054024 (2016).

[59] L. Gamberg, A. Metz, D. Pitonyak, and A. Prokudin, Phys. Lett. B 781, 443 (2018).

[60] I. Scimemi, A. Tarasov, and A. Vladimirov, J. High Energy Phys. 05 (2019) 125.

[61] D. Boer, P. J. Mulders, and F. Pijlman, Nucl. Phys. B667, 201 (2003).

[62] D. W. Sivers, Phys. Rev. D 41, 83 (1990).

[63] D. W. Sivers, Phys. Rev. D 43, 261 (1991).

[64] J.-W. Qiu, T. C. Rogers, and B. Wang, Phys. Rev. D 101, 116017 (2020).

[65] A. Airapetian et al. (HERMES Collaboration), Phys. Rev. Lett. 103, 152002 (2009).

[66] M. Alekseev et al. (COMPASS Collaboration), Phys. Lett. B 673, 127 (2009).

[67] X. Qian et al. (The Jefferson Lab Hall A Collaboration), Phys. Rev. Lett. 107, 072003 (2011).

[68] C. Adolph et al. (COMPASS Collaboration), Phys. Lett. B 744, 250 (2015).

[69] Y.X. Zhao et al. (Jefferson Lab Hall A Collaboration), Phys. Rev. C 90, 055201 (2014).

[70] C. Adolph et al. (COMPASS Collaboration), Phys. Lett. B 770, 138 (2017).

[71] A. Airapetian et al. (HERMES Collaboration), Phys. Lett. B 693, 11 (2010).

[72] L. Adamczyk et al. (STAR Collaboration), Phys. Rev. Lett. 116, 132301 (2016).

[73] M. Aghasyan et al. (COMPASS Collaboration), Phys. Rev. Lett. 119, 112002 (2017).

[74] R. Seidl et al. (Belle Collaboration), Phys. Rev. D 78, 032011 (2008).

[75] J. P. Lees et al. (BABAR Collaboration), Phys. Rev. D 90, 052003 (2014).

[76] J. P. Lees et al. (BABAR Collaboration), Phys. Rev. D 92 , 111101 (2015).
[77] M. Ablikim et al. (BESIII Collaboration), Phys. Rev. Lett. 116, 042001 (2016).

[78] H. Li et al. (Belle Collaboration), Phys. Rev. D 100, 092008 (2019).

[79] J. P. Ralston and D. E. Soper, Nucl. Phys. B152, 109 (1979).

[80] J. C. Collins, Nucl. Phys. B396, 161 (1993).

[81] A. Bacchetta and A. Prokudin, Nucl. Phys. B875, 536 (2013).

[82] Z.-B. Kang, A. Prokudin, P. Sun, and F. Yuan, Phys. Rev. D 93, 014009 (2016).

[83] K. Kanazawa, Y. Koike, A. Metz, and D. Pitonyak, Phys. Rev. D 89, 111501(R) (2014).

[84] L. Gamberg, Z.-B. Kang, D. Pitonyak, and A. Prokudin, Phys. Lett. B 770, 242 (2017).

[85] M. Anselmino, M. Boglione, U. D’Alesio, A. Kotzinian, F. Murgia, and A. Prokudin, Phys. Rev. D 71, 074006 (2005).

[86] M. Anselmino, D. Boer, U. D’Alesio, and F. Murgia, Phys. Rev. D 63, 054029 (2001).

[87] M. Anselmino, M. Boglione, U. D’Alesio, A. Kotzinian, F. Murgia, and A. Prokudin, Phys. Rev. D 72, 094007 (2005).

[88] W. Vogelsang and F. Yuan, Phys. Rev. D 72, 054028 (2005).

[89] J. Collins, A. Efremov, K. Goeke, S. Menzel, A. Metz, and P. Schweitzer, Phys. Rev. D 73, 014021 (2006).

[90] J. C. Collins et al., arXiv:hep-ph/0511272.

[91] M. Anselmino, M. Boglione, U. D’ Alesio, A. Kotzinian, F. Murgia, A. Prokudin, and C. Türk, Phys. Rev. D 75, 054032 (2007).

[92] M. Anselmino, M. Boglione, U. D’Alesio, A. Kotzinian, F. Murgia, A. Prokudin, and S. Melis, Nucl. Phys. B, Proc. Suppl. 191, 98 (2009).

[93] P. Schweitzer, T. Teckentrup, and A. Metz, Phys. Rev. D 81, 094019 (2010).

[94] J.-W. Qiu, M. Schlegel, and W. Vogelsang, Phys. Rev. Lett. 107, 062001 (2011).

[95] M. Anselmino, M. Boglione, U. D'Alesio, S. Melis, F. Murgia, and A. Prokudin, Phys. Rev. D 87, 094019 (2013).

[96] A. Signori, A. Bacchetta, M. Radici, and G. Schnell, J. High Energy Phys. 11 (2013) 194.

[97] M. Anselmino, M. Boglione, J. O. Gonzalez Hernandez, S. Melis, and A. Prokudin, J. High Energy Phys. 04 (2014) 005.

[98] D. Boer and W. J. den Dunnen, Nucl. Phys. B886, 421 (2014).

[99] U. D'Alesio, F. Murgia, and M. Zaccheddu, arXiv: 2003.01128.

[100] D. Callos, Z.-B. Kang, and J. Terry, arXiv:2003.04828.

[101] K. Orginos, A. Radyushkin, J. Karpie, and S. Zafeiropoulos, Phys. Rev. D 96, 094503 (2017).

[102] M. Anselmino, M. Boglione, U. D’Alesio, F. Murgia, and A. Prokudin, J. High Energy Phys. 04 (2017) 046.

[103] M. Anselmino, M. Boglione, U. D’Alesio, J. O. G. Hernandez, S. Melis, F. Murgia, and A. Prokudin, Phys. Rev. D 92, 114023 (2015).

[104] P. Sun and F. Yuan, Phys. Rev. D 88, 034016 (2013).

[105] Z.-B. Kang, A. Prokudin, P. Sun, and F. Yuan, Phys. Rev. D 91, 071501 (2015).

[106] M. G. Echevarria, A. Idilbi, Z.-B. Kang, and I. Vitev, Phys. Rev. D 89, 074013 (2014). 
[107] Z.-B. Kang, A. Prokudin, F. Ringer, and F. Yuan, Phys. Lett. B 774, 635 (2017).

[108] D. W. Duke and J. F. Owens, Phys. Rev. D 30, 49 (1984).

[109] A. Accardi, L. T. Brady, W. Melnitchouk, J. F. Owens, and N. Sato, Phys. Rev. D 93, 114017 (2016).

[110] D. de Florian, R. Sassot, and M. Stratmann, Phys. Rev. D 76, 074033 (2007).

[111] P. C. Barry, N. Sato, W. Melnitchouk, and C.-R. Ji, Phys. Rev. Lett. 121, 152001 (2018).

[112] The precision of the COMPASS Drell-Yan data is such that using next-to-leading-order pion PDFs will not affect our results.

[113] G. Schnell (HERMES Collaboration), Proc. Sci., DIS2010 (2010) 247.

[114] B. Parsamyan, Proc. Sci., DIS2013 (2013) 231 [arXiv: 1307.0183].

[115] U. D’Alesio, C. Flore, and A. Prokudin, Phys. Lett. B 803, 135347 (2020).

[116] A. Airapetian et al. (HERMES), Phys. Rev. D 87, 074029 (2013).

[117] N. Sato, C. Andres, J. J. Ethier, and W. Melnitchouk (JAM Collaboration), Phys. Rev. D 101, 074020 (2020).
[118] https://github.com/QCDHUB/jam3dlib.

[119] M. Radici and A. Bacchetta, Phys. Rev. Lett. 120, 192001 (2018).

[120] J. Benel, A. Courtoy, and R. Ferro-Hernandez, Eur. Phys. J. C 80, 465 (2020).

[121] R. Gupta, Y.-C. Jang, B. Yoon, H.-W. Lin, V. Cirigliano, and T. Bhattacharya, Phys. Rev. D 98, 034503 (2018).

[122] N. Hasan, J. Green, S. Meinel, M. Engelhardt, S. Krieg, J. Negele, A. Pochinsky, and S. Syritsyn, Phys. Rev. D 99, 114505 (2019).

[123] C. Alexandrou, S. Bacchio, M. Constantinou, J. Finkenrath, K. Hadjiyiannakou, K. Jansen, G. Koutsou, and A. Vaquero Aviles-Casco, arXiv:1909.00485.

[124] G. R. Goldstein, J. O. Gonzalez Hernandez, and S. Liuti, arXiv:1401.0438.

[125] M. Radici, A. Courtoy, A. Bacchetta, and M. Guagnelli, J. High Energy Phys. 05 (2015) 123.

[126] M. Pitschmann, C.-Y. Seng, C. D. Roberts, and S. M. Schmidt, Phys. Rev. D 91, 074004 (2015).

[127] J. Chen, H. Gao, T. Hemmick, Z. E. Meziani, and P. Souder (SoLID Collaboration), arXiv:1409.7741. 